\title{
Incentive Contract between Banks and B2B Platform in Online Agricultural Product Supply Chain Finance
}

\author{
Xiaoxu Chen ${ }^{1}$, Peng $\mathrm{Xu}^{2,3} \&$ Guoqiang Yang ${ }^{4}$ \\ ${ }^{1}$ School of Economic and Management, Shanxi University, Taiyuan 030006, China \\ ${ }^{2}$ School of Business, Southwest University of Political Science and Law, Chongqing 401120, China \\ ${ }_{3}^{3}$ John Molson School of Business Concordia University, Montreal H3G 1M8, Canada \\ ${ }^{4}$ Department of Medical Imaging, Shanxi Medical University, Taiyuan 030001, China \\ Correspondence: Peng Xu, School of Business, Southwest University of Political Science and Law, Yubei District, \\ Chongqing 401120, China. E-mail: xupengswul@163.com
}

Received: March 8, 2019

Accepted: June 10, 2019

Online Published: June 20, 2019

doi:10.5430/bmr.v8n2p20

URL: https://doi.org/10.5430/bmr.v8n2p20

\begin{abstract}
Online agricultural supply chain finance, as an effective way to solve the financing difficulties of small and medium-sized agricultural enterprises in the chain, has made rapid progress in recent years, and the cooperation between banks and e-commerce has become the mainstream mode. Taking the electronic order pledge of agricultural products as an example, this paper discusses the incentive contract design between banks and B2B platform from the perspective of moral hazard prevention and the use of principal-agent theory. In this paper, the principal-agent model is constructed by considering the bank's effort and no effort, and then give the optimal incentive coefficient and fixed return. The results show that banks' effort will increase the level of efforts of B2B platform, but also increase their own variable payment; under the given conditions, the bank's effort will increase its income, and at a certain level of effort, the largest increase in revenue, In addition, improving the application level of B2B data processing technology and the degree of data pledge development will contribute to the increase of incentive coefficient and revenue.
\end{abstract}

Keywords: online, agricultural supply chain finance, incentive contract, B2B platform

\section{Introduction}

With the rapid development and maturity of internet technology, the e-commerce business of agricultural products is developing rapidly. Many small and medium-sized agricultural enterprises and core enterprises in the supply chain trade on the B2B platform. Upstream farmers, cooperatives and small and medium-sized enterprises have a large number of electronic orders from the core enterprises. However, the upstream suppliers lack enough funds to purchase raw material for production. They are unable to produce normally, resulting in the failure to perform the contract on time. Therefore, it is an urgent task to solve the shortage of funds. The agricultural order financing provides an effective way to alleviate the financing dilemma. Because of the huge advantages of business modes, it has attracted wide attention. Kaplan and Sawhney studied the path and method of an electronic center introduced into the order financing (Kaplan S, Sawhney M., 2000). Heng analyzed the impact of the e-commerce on the financial system, and gave the concrete manifestation of the interaction between the two sides from three aspects (Heng M. S. H., 2001). Gu Min studied the specific modes of the electronic order financing according to its characteristics (Guo J.E., Shi J.Z., Wang Z. X., 2014). Li analyzed the difference of risk elements between online and offline order financing in detail, and put forward the precautions against the financial risk of the online order financing (Gu M., 2015). Guo et al. studied risk factors and management countermeasures of the online supply chain finance (Li Y.Q., 2015). Shi et al. discussed the incentive contract design between a bank and a B2B e-commerce platform from two aspects of joint credit and entrusted credit (Shi J. Z, Guo J. E., 2015) (Shi J. Z., 2015). Wang et al. studied the evolution process of the principal-agent problem between the bank and the B2B platform, and analyzed the incentive contract design considering fairness preference factors (Wang K. F., Shi K. R., 2016)(Wang K. F, Shi K. R.,, 2017). $\mathrm{Xu}$ studied the construction of a risk index evaluation system of the online agricultural supply chain finance, and evaluated risks in the business applying for analytic hierarchy process and structural equation model (Xu P., 2016).

However, compared with the traditional order financing business, the significant feature of the agricultural supply chain finance is the participation of the B2B platform, and in the process of business development, the mainstream 
mode of cooperation between the bank and the B2B platform is gradually formed because of the respective advantages of both sides (Guo J.E., Shi J.Z., Wang Z. X., 2014). The bank has scientific risk assessment mechanism, risk prevention mechanism, strong financial strength et al. The B2B platform is outstanding in data collection, data processing, big data application, information sharing et al. With the development of science and technology, the two sides have made use of their respective advantages to cooperate to complete the business, which is in line with the interests of both sides. So in recent years, the practical business based on the cooperation between the two sides has developed rapidly (Gu M., 2015).

To the best of our knowledge, there is little literature on contract design between the bank and the B2B platform. We fail to find a paper on moral hazard of the B2B platform during the course of their cooperation, this paper may be the first to discuss the question in agriculture supply chain finance, and to give the equilibrium results of banks.

\section{Assumptions}

(1)Assuming that banks authorizes B2B platforms to examine financing customers, and work efficiency and effect of B2B platforms will affect banks' earnings.

(2)Based on references (Xu P., 2016), assuming that the utility function of B2B Platform is linear, the specific expression is as follows: $\pi=\kappa a+\omega+\xi$

Where, $a$ denotes effort level of B2B platforms, the more efforts they make, the greater the probability of successful repayment of financing customers, the more the utility is. $\kappa$ denotes the examination efficiency of B2B platforms, and $\kappa>0$, it is highly related to their large data processing technology, experience, relationship with financing enterprises, knowledge of core enterprises etc. The greater $\kappa$ is, the more the output income of B2B platform is.

Based on the efforts of B2B platforms, the contract designed by banks is as follows.

$$
\phi=\eta+\beta[\pi(a)-\pi(0)]
$$

Wherein, $\eta$ denotes fixed reward paid to B2B platforms for banks, $\beta$ denotes incentive coefficient, which means allocation proportion of output income, $\pi(a)-\pi(0)$ is output income level when B2B platforms choose to work hard, so $\pi(a)-\pi(0)>0$, when B2B platforms does not make any effort, that is, $a=0$, so $\pi(a)-\pi(0)=0$, B2B platforms only obtain the fixed rewards.

The higher the effort level of B2B platforms, the more the costs occur. Assuming that the efforts cost made by B2B platforms are expressed as follow.

$$
C_{t}=\frac{\lambda a^{2}}{2}
$$

Wherein, $\lambda$ denotes work effectiveness, it is related to work experience, large data processing technology, relationship with financing enterprises, knowledge of core enterprises etc. The smaller $\lambda$, the less the cost is.

Assuming that a bank is risk neutral, so its expected utility is equal to its deterministic equivalent income:

$$
E\left(R_{b}\right)=\pi-\phi=(1-\beta) \kappa a+\omega-\eta
$$

Assuming that $\mathrm{B} 2 \mathrm{~B}$ is risk-averse and risk-averse is $\rho$, the random net profits of $\mathrm{B} 2 \mathrm{~B}$ platforms is:

$$
R=\phi-C_{t}=\beta \kappa a+\eta-\frac{\lambda a^{2}}{2}
$$

The deterministic equivalent income of B2B platforms is:

$$
E\left(R_{t}\right)=\beta \kappa a+\eta-\frac{\lambda a^{2}}{2}-\frac{\rho \beta^{2} \sigma^{2}}{2}
$$




\section{Incentive Contracts in two Cases}

\subsection{Incentive Contracts without Banks' Effort}

Under information asymmetry, banks design contracts to make B2B platforms work hard when they examine financing customers. The purpose is to maximize their profits. The realization of this goal is restricted by two conditions: participation constraint and incentive compatibility constraint (Guo Z Y, Luo Y X T., 2017). In other words, under these two conditions, banks achieve most income by choosing fixed payment and incentive coefficient. The model is expressed as follows.

$$
\begin{gathered}
\max E\left(R_{b}\right) \\
\text { s.t. }(I R) \beta \kappa a+\eta-\frac{\lambda a^{2}}{2}-\frac{\rho \beta^{2} \sigma^{2}}{2} \geq v_{0} \\
\left.(I C) \max \beta \kappa a+\eta-\frac{\lambda a^{2}}{2}-\frac{\rho \beta^{2} \sigma^{2}}{2}\right]
\end{gathered}
$$

Where, $v_{0}$ denotes retained earnings of B2B platform, By Eq. (3), the optimal effort level of B2B platforms is as follows:

$$
a^{*}=\frac{\beta \kappa}{\lambda}
$$

In the case of equilibrium, the income of B2B platforms will not exceed their retained earnings, that is, the deterministic equivalent earnings of $\mathrm{B} 2 \mathrm{~B}$ platforms equals their retained earnings, $\beta \kappa a+\eta-\frac{\lambda a^{2}}{2}-\frac{\rho \beta^{2} \sigma^{2}}{2}=v_{0}$. From this, we can see that the fixed payment expression of banks is as follows:

$$
\eta=v_{0}-\beta \kappa a+\frac{\lambda a^{2}}{2}+\frac{\rho \beta^{2} \sigma^{2}}{2}
$$

Substituting Eq.(4) and Eq.(5)into Eq.(1), and solving the first-order condition $E\left(R_{b}\right) / \beta=0$ for $\beta$ gives best incentive coefficient as.

$$
\beta^{*}=\frac{\kappa^{2}}{\kappa^{2}+\lambda \rho \sigma^{2}}
$$

Substituting Eq.(4) and Eq.(6)into Eq.(5), The best fixed payment is:

$$
\eta^{*}=v_{0}+\frac{\kappa^{6}}{2 \lambda\left[\kappa^{2}+\lambda \rho \sigma^{2}\right]}-\frac{\kappa^{4} \lambda \rho \sigma^{2}}{2 \lambda\left[\kappa^{2}+\lambda \rho \sigma^{2}\right]}
$$

Substituting Eq.(4), Eq.(5)and Eq.(6) into Eq.(1), the expected return of the bank is:

$$
E\left(R_{b}^{*}\right)=\omega-v_{0}+\frac{\kappa^{4}}{2 \lambda\left[\kappa^{2}+\lambda \rho \sigma^{2}\right]}
$$

\subsection{Incentive Contracts with Banks' Effort}

During the course of examination, banks give B2B platforms help in some activates, such as business training, review guidance, risk control, information security, financial statement analysis, etc. Banks have an advantage in these activities. By transferring knowledge and skills to B2B platform, it is undoubtedly of great significance to improve the efficiency of review, and then to improve the output income.

With banks' effort, the output function of B2B platforms is as follows:

$$
\pi=(\kappa+\theta) a+\omega+\xi
$$


Where, $\theta$ denotes increase in efficiency of examination with banks' effort, which is related to the efforts level of banks. Assuming that the corresponding cost of bank's effort is $C_{b}=\frac{\mu e^{2}}{2}, e$ denotes the effort level of banks, $\mu$ denotes the effectiveness of bank's efforts.

Based on the above analysis, the expected income of banks is:

$$
E\left(R_{b}\right)=\pi-\phi=(1-\beta)(\kappa+\theta) a+\omega-\eta-\frac{\mu e^{2}}{2}
$$

Based on the above analysis, the deterministic equivalent income of B2B platforms is:

$$
E\left(R_{t}\right)=\beta(\kappa+\theta) a+\eta-\frac{\lambda a^{2}}{2}-\frac{\rho \beta^{2} \sigma^{2}}{2}
$$

Combining the above analysis, the optimal incentive contract design model with bank's effort is as follow.

$$
\begin{gathered}
\max E\left(R_{b}\right) \\
\text { s.t.(IR) } \beta(\kappa+\theta) a+\eta-\frac{\lambda a^{2}}{2}-\frac{\rho \beta^{2} \sigma^{2}}{2} \geq v_{0} \\
(\text { IC }) \max \left[\beta(\kappa+\theta) a+\eta-\frac{\lambda a^{2}}{2}-\frac{\rho \beta^{2} \sigma^{2}}{2}\right]
\end{gathered}
$$

By solving the model, we get the equilibrium solution of the incentive contract.

$$
\begin{gathered}
a^{*}=\frac{\beta(\kappa+\theta)}{\lambda} \\
\beta^{*}=\frac{(\kappa+\theta)^{2}}{(\kappa+\theta)^{2}+\lambda \rho \sigma^{2}} \\
\eta^{*}=v_{0}+\frac{(\kappa+\theta)^{6}}{2 \lambda\left[(\kappa+\theta)^{2}+\lambda \rho \sigma^{2}\right]}-\frac{(\kappa+\theta)^{4} \lambda \rho \sigma^{2}}{2 \lambda\left[(\kappa+\theta)^{2}+\lambda \rho \sigma^{2}\right]} \\
E\left(R_{\delta}^{*}\right)=\omega-\gamma+\frac{(\kappa+\theta)^{4}}{2 \lambda\left[(\kappa+\theta)^{2}+\lambda \rho \sigma^{2}\right]}-\frac{\mu e^{2}}{2}
\end{gathered}
$$

\subsection{Comparative Analysis of Equilibrium Results in Two Cases}

We obtain some new findings through the comparative analysis of these two cases

Proposition 1: With banks' efforts, the effort level of B2B platform increase.

The difference of effort level of B2B platforms in two cases is as follows:

$$
\Delta a=a^{* *}-a^{*}=\frac{\beta(\kappa+\theta)}{\lambda}-\frac{\beta \kappa}{\lambda}=\frac{\beta \theta}{\lambda}>0
$$

From (16), we see that B2B platform will work harder during the course of completing the entrusted task under banks' effort.

Proposition 2: With banks' efforts, the incentive coefficient increases.

The difference of incentive coefficient in two cases is as follows:

$$
\Delta \beta=\beta^{* *}-\beta^{*}=\frac{(\kappa+\theta)^{2}}{(\kappa+\theta)^{2}+\lambda \rho \sigma^{2}}-\frac{\kappa^{2}}{\kappa^{2}+\lambda \rho \sigma^{2}}=\frac{1}{1+\frac{\lambda \rho \sigma^{2}}{(\kappa+\theta)^{2}}}-\frac{1}{1+\frac{\lambda \rho \sigma^{2}}{\kappa^{2}}}>0
$$


From (17), we see that banks will give B2B platforms more incentive rewards under banks' effort.

Proposition 3: With banks' effort, when $\varphi>\frac{\mu e^{2}}{2}, \Delta E\left(R_{b}\right)>0$, that is, banks' profits increase, where, $\varphi=\frac{(\kappa+\theta)^{4}}{2 \lambda\left[(\kappa+\theta)^{2}+\lambda \rho \sigma^{2}\right]}-\frac{\kappa^{4}}{2 \lambda\left[\kappa^{2}+\lambda \rho \sigma^{2}\right]}>0$.

The difference of the incentive coefficients in two cases is as follows:

$$
\Delta E\left(R_{b}\right)=E^{*}\left(R_{b}\right)-E^{*}\left(R_{b}\right)=\left[\frac{(\kappa+\theta)^{4}}{2 \lambda\left[(\kappa+\theta)^{2}+\lambda \rho \sigma^{2}\right]}-\frac{\kappa^{4}}{2 \lambda\left[\kappa^{2}+\lambda \rho \sigma^{2}\right]}\right]-\frac{\mu e^{2}}{2}
$$

Banks provide some help in activities, such as financial indicators analysis, risk control methods, review procedure design, etc. In these activities, banks are very experienced. In other words, the effectiveness of efforts will be high, so the value $\mu$ will be small. When $\mu \rightarrow 0, \frac{\mu e^{2}}{2} \rightarrow 0$, so $\varphi>\frac{\mu e^{2}}{2}$ is satisfied. As a result, banks' profits increase.

Proposition 4: When $\frac{\lambda}{\kappa^{2}}$ decreases, both banks' incentive coefficient and profits increase.

By (6), then $\beta^{*}=\frac{1}{1+\frac{\lambda}{\kappa^{2}} \rho \sigma^{2}}$. When $\frac{\lambda}{\kappa^{2}} \quad$ decreases, $\beta^{*}$ increases. By (8), then $E\left(R_{b}^{*}\right)=\omega-v_{0}+\frac{1}{\left[\lambda(\lambda)^{2}\right]}$ We see that banks' profits increase with the decrease of the value $\frac{\lambda}{\kappa^{2}}$. $2\left[\frac{\lambda}{\kappa^{2}}+\left(\frac{\lambda}{\kappa^{2}}\right)^{2} \rho \sigma^{2}\right]$

With the deep application of big data technology and data pledge technology, and the "three streams" will be highly integrated. In this case, the work efficiency and effectiveness of B2B platform will be enhanced, that is, the value $\kappa$ will be larger, the value $\lambda$ will be smaller, the value $\frac{\lambda}{\kappa^{2}}$ will be significantly smaller. Although banks' incentive coefficient increases, their profits still increase.

\section{Numerical Examples}

To further verify the feasibility and validity of research conclusions, the paper gives one example. Our example is generated as follow, the function between $\theta$ and $e$ is given by $e=4 \theta$,other parameters are given by $\kappa=2$, $\theta=1, \lambda=0.5, \quad \mu=0.2, \rho=1, \omega=15, v_{0}=12, \sigma^{2} \sim(0,36)$. The optimal effort level, banks' optimal incentive coefficient and profits in two cases are listed in table1

Table 1. Optimal effort level, banks' optimal incentive coefficient and profits in two cases

\begin{tabular}{lllll}
\hline Var. & Without effort & With effort & Comparison & \\
\hline$a$ & 0.728 & 1.998 & $\Delta a$ & 1.270 \\
\hline$\beta$ & 0.182 & 0.333 & $\Delta \beta$ & 0.151 \\
\hline $\boldsymbol{E}\left(\boldsymbol{R}_{b}\right)$ & 2.127 & 4.400 & $\Delta E\left(\boldsymbol{R}_{b}\right)$ & 2.273 \\
\hline
\end{tabular}

With banks' efforts, the effort level of B2B platforms, banks' incentive coefficient and profits vary with their efforts. The results are shown in table 2. 
Table 2. Changes of equilibrium solution with banks' effort

\begin{tabular}{lllllll}
\hline Var. & $e=1$ & $e=2$ & $e=4$ & $e=6$ & $e=8$ & $e=10$ \\
\hline$a$ & 0.99 & 1.29 & 1.998 & 2.870 & 3.592 & 4.761 \\
\hline$\Delta a$ & 0.262 & 0.562 & 1.270 & 2.142 & 2.864 & 4.033 \\
\hline$\beta$ & 0.220 & 0.258 & 0.333 & 0.410 & 0.449 & 0.529 \\
\hline$\Delta \beta$ & 0.038 & 0.076 & 0.151 & 0.228 & 0.267 & 0.341 \\
\hline$E\left(R_{b}\right)$ & 4.003 & 4.210 & 4.400 & 4.350 & 4.119 & 3.753 \\
\hline$\Delta E\left(R_{b}\right)$ & 1.876 & 2.083 & 2.273 & 2.233 & 1.992 & 1.626
\end{tabular}

From the above figures, we see that the efforts level of B2B platforms and banks' incentive coefficient will increase with increase of banks' effort level. Within a certain range, the profits will increase with the increase of banks' effort level. When banks' effort level reaches a certain value, their profits will maximize, and then decrease with the increase of their efforts.

To further verify the change of banks' incentive coefficients and profits with $\frac{\lambda}{\kappa^{2}}$, six groups of data of $\lambda$ and $\kappa^{2}$ are given by $(0.6,1),(0.5,2),(0.4,3),(0.3,4),(0.2,5),(0.1,6)$. The results are shown in Table 3 .

Table 3. Change of both banks 'incentive coefficient and profits with $\frac{\lambda}{\kappa^{2}}$

\begin{tabular}{lllllll}
\hline \multicolumn{1}{rl}{ Var. } & $(0.6,1)$ & $(0.5,2)$ & $(0.4,3)$ & $(0.3,4)$ & $(0.2,5)$ & $(0.1,6)$ \\
\hline$\beta$ & & & & & & \\
\hline$E\left(R_{b}\right)$ & 0.04 & 0.18 & 0.39 & 0.59 & 0.81 & 0.90 \\
\hline
\end{tabular}

From Table 3, we can see that both banks' incentive coefficient and profits increase with the decrease of $\frac{\lambda}{\kappa^{2}}$, and the growth rate is from rapid growth to slow growth, among which the changes of banks profits are most obvious in the two groups of data $(0.3,4)$ and $(0.2,5)$. The change in value $\frac{\lambda}{\kappa^{2}}$ is closely related to the application level of large data processing technology, the development of pledged data, work experience and the aggregation of "three streams" (logistics, information flow, capital flow) of B2B platforms.

\section{Conclusions}

In recent years, with the rapid development of internet technology, the integration of "internet+" traditional industries is getting deeper and deeper. On-line supply chain finance of agricultural products is a brand-new financing mode promoted by supply chain finance with the help of internet technology. This mode overcomes the limitations of traditional finance mode. It provides a path for small and medium-sized agricultural enterprises to achieve loans from banks. But on-line agricultural products supply chain finance is a complex business type with multi-subjects, multi-procedures and multi-links. It faces many risks, especially the principal-agent problem, which is one of the key issues. Based on this, this paper uses principal-agent theory to study the contract design. The results show that banks' efforts will improve effort level of B2B platforms, and increase their profits under certain conditions.

\section{Acknowledgements}

This work was supported by the Shanxi Soft Science Project(no.2018041044-1), the National Social Science Foundation of China (no.16BGL002) and Shanxi Philosophy and Social Sciences Project of Universities(no.201803048).

\section{References}

$\mathrm{Gu}$ M. (2015). Research on supply chain finance model of e-commerce enterprises. Journal of Commercial Economics, (18), 86-88. https://doi.org/10.3969/j.issn.1002-5863.2015.18.035

Guo J.E., Shi J.Z., Wang Z. X. (2014). Research on the mode evolution and risk management of the online supply chain finance based on the third-party B2B e-commerce platform. Journal of Business Economics, (1), 13-22. https://doi.org/10.15896/j.xjtuskxb.201504002 
Guo Z Y, Luo Y X T. (2017). Credit constraint exports in countries with different degrees of contract enforcement. Business and Economic Research, 7(1), 227-241. https://doi.org/10.5296/ber.v7i1.10923

Heng M. S. H. (2001). Implications of E-commerce for Banking and Finance. Netherlands: Vrije University, 1-14. https://doi.org/10.1007/0-306-47009-8_22

Kaplan S, Sawhney M. (2000). E-hubs: the new B2B market places. Harvard business review, 78(3), 97-106. https://doi.org/10.1210/jc.2009-0402

Li Y.Q. (2015). B2B platform online supply chain financial risk identification and prevention. Journal of Commercial Economics, (19), 89-90. https://doi.org/10.3969/j.issn.1002-5863.2015.18.036

Shi J. Z, Guo J. E. (2015). Study on the development and domestic practice of supply chain finance from the perspective of the internet. Journal of $\mathrm{Xi}^{\prime}{ }^{\prime}$ an Jiaotong University: social sciences, 35(4), 10-16. https://doi.org/10.15896/j.xjtuskxb.201504002

Shi J. Z. (2015). Study on the incentive contract between banks and B2B platforms based on the online supply chain finance. Journal of Management Science, 28(5), 79-92. https://doi.org/10.3969/j.issn.1672-0334.2015.05.007

Wang K. F, Shi K. R. (2017). Bank and online supply chain finance evolution analysis of principal agent in B2B platform. Financial theory and practice, (5), 73-77. https://doi.org/10.3969/j.issn.1003-4625.2017.05.013

Wang K. F., Shi K. R. (2016). Research on incentive mechanism of banks to B2B platform based on fairness preference. Financial theory and practice, (10), 62-66. https://doi.org/10.3969/j.issn.1003-4625.2016.10.012

Xu P. (2016). Risk prevention of online agricultural product supply chain finance. Journal of south China agricultural university: social sciences, 15(6), 93-103. https://doi.org/10.7671/j.issn.1672-0202.2016.06.010

$\mathrm{Xu}$ P. (2016). Risk prevention of online agricultural product supply chain finance based on factor analysis perspective. Journal of agro-forestry economics and management, 15(6), 674-680. https://doi.org/10.16195/j.cnki.cn36-1328/f.2016.06.083 